\title{
ON MINAKSHISUNDARAM-PLEIJEL ZETA FUNCTIONS OF SPHERES
}

\author{
E. CARLETTI AND G. MONTI BRAGADIN
}

(Communicated by Dennis A. Hejhal)

ABSTRACT. The aim of this paper is to show that the Minakshisundaram-Pleijel zeta function $Z_{k}(s)$ of $k$-dimensional sphere $S^{k}, k \geq 2$ (defined in $\Re e(s)>\frac{k}{2}$ by

$$
Z_{k}(s)=\sum_{n=1}^{\infty} \frac{P_{k}(n)}{[n(n+k-1)]^{s}}
$$

with $(k-1) ! P_{k}(n)=\mathscr{R}(n+1, k-2)(2 n+k-1)$ where the "rising factorial" $\mathscr{R}(x, n)=x(x+1) \cdots(x+n-1)$ is defined for real number $x$ and $n$ nonnegative integer) can be put in the form

$$
(k-1) ! Z_{k}(s)=\sum_{l=0}^{\infty}(-1)^{l}\left(\frac{k-1}{2}\right)^{2 l}\left(\begin{array}{c}
-s \\
l
\end{array}\right) \sum_{j=0}^{k-1} B_{k, j} \zeta\left(2 s+2 l-j, \frac{k+1}{2}\right)
$$

where $B_{k, j}$ are explicitly computed. The above formula allows us to find explicitly the residue of $Z_{k}(s)$ at the pole $s=\frac{k}{2}-n, n \in \mathrm{N}$,

$$
\frac{1}{(k-1) !} \sum_{h=0}^{\frac{k}{2}-1} \sum_{\substack{l+h=n \\
l \geq 0}}(-1)^{l}\left(\frac{k-1}{2}\right)^{2 l}\left(\begin{array}{c}
n-\frac{k}{2} \\
l
\end{array}\right) B_{k, k-2 h-1} .
$$

In passing, we also obtain apparently new relations among the Stirling numbers.

\section{INTRODUCTION AND NOTATION}

In 1949, Minakshisundaram and Pleijel introduced a generalized Dirichlet series associated with a compact riemannian manifold ([5]). It is a standard result that the eigenvalues of the Laplace operator acting on $L^{2}(M)$ form a sequence

$$
0=\lambda_{0}<\lambda_{1} \leq \cdots \leq \lambda_{n} \leq \cdots
$$

where every $\lambda_{i}$ is repeated with its multiplicity which is finite. The Minakshisundaram-Pleijel zeta function of the riemannian manifold $M$ is defined in

Received by the editors March 24, 1993.

1991 Mathematics Subject Classification. Primary 11M41. 
$\Re e(s)>\frac{\operatorname{dim} M}{2}$ by

$$
\zeta_{M}(s)=\sum_{n=1}^{\infty} \lambda_{n}^{-s} .
$$

This function is closely connected with the problem of the asymptotic behaviour of the eigenvalues. Minakshisundaram and Pleijel proved the meromorphic continuation of $\zeta_{M}(s)$ by using tools of global analysis; more precisely they proved that $\zeta_{M}(s)$ is analytic on $\mathbb{C}$ except at simple poles $s=\frac{\operatorname{dim} M}{2}-k, k \in$ $\mathbb{N}$, and expressed the residues in terms of metric invariants of $M$ in a quite involved manner so that they are not at all efficiently computable even if the eigenvalues are explicitly known (f.i. $k$-dimensional sphere or the projective real and complex spaces equipped with the standard metric) (see [1] and [3]).

In this note we express $\zeta_{M}(s)$, when $M=\mathbb{S}^{k}$ or $\mathbb{P}^{k}(\mathbb{R})$, in a convenient way, which allows us to write down the residues explicitly.

The Minakshisundaram-Pleijel zeta function $Z_{k}(s)$ of $k$-dimensional sphere $S^{k}$ takes the form, in $\Re e(s)>\frac{k}{2}$

$$
Z_{k}(s)=\sum_{n=1}^{\infty} \frac{P_{k}(n)}{[n(n+k-1)]^{s}}
$$

with $(k-1) ! P_{k}(n)=\mathscr{R}(n+1, k-2)(2 n+k-1)$ (see [1]) where the "rising factorial" $\mathscr{R}(x, n)=x(x+1) \cdots(x+n-1)$ is defined for real number $x$ and $n$ non-negative integer. We observe that if $k=1$, the MinakshisundaramPleijel zeta function reduces to $2 \zeta(2 s)$ where $\zeta(s)$ is the Riemann zeta function.

\section{STIRLING NUMBERS}

We define the Stirling numbers of the first kind $s_{n, k}$ by

$$
\mathscr{R}(x, n)=\sum_{k=0}^{n}(-1)^{n+k} s_{n, k} x^{k} .
$$

We list some useful formulas concerning Stirling numbers here (see [4], Chapter IV, where the Stirling numbers are denoted by $S_{n}^{k}$ )

$$
\begin{gathered}
s_{n+1, k}=s_{n, k-1}-n s_{n, k} \quad(\text { see [4], } 5 \text { of p. 143), } \\
s_{n, k}=\sum_{j=0}^{n}\left(\begin{array}{l}
j \\
k
\end{array}\right) s_{n+1, j+1} \quad(\text { see [4], 4. of p. 187), } \\
(-1)^{k} s_{n+1, k+1}=\sum_{j=0}^{n}(-1)^{j}\left(\begin{array}{l}
j \\
k
\end{array}\right) s_{n, j} \quad(\text { see [4], 3. of p. 186), } \\
s_{n+1, k}=\sum_{j=0}^{n}(-1)^{j} \frac{n !}{(n-j) !} s_{n-j, k-1} .
\end{gathered}
$$


If we put

$$
(k-1) ! P_{k}(n)=\sum_{m=0}^{k-1} \alpha_{k, m} n^{m}
$$

we also have

$$
\alpha_{k, m}=(-1)^{m+k+1}\left(s_{k, m+1}+s_{k-1, m}\right), \quad 0 \leq m \leq k-1 .
$$

Equation (3) follows from (1a) and (1c).

\section{Statement of Results}

We get the following results

Theorem 1. Let us consider

$$
L_{k}(s)=\sum_{n=1}^{\infty} \frac{P_{k}(n)}{\left[\left(n+\delta_{1}\right)\left(n+\delta_{2}\right)\right]^{s}}
$$

where $\delta_{1} \leq \delta_{2}$ are non-negative real numbers with $\delta_{1}+\delta_{2}=k-1$ and $P_{k}(n)$ is as above. If $k \geq 2$, then

$$
(k-1) ! L_{k}(s)=\sum_{l=0}^{\infty}(-1)^{l}\left(\frac{\delta_{2}-\delta_{1}}{2}\right)^{2 l}\left(\begin{array}{c}
-s \\
l
\end{array}\right) \sum_{j=0}^{k-1} B_{k, j} \zeta\left(2 s+2 l-j, \frac{k+1}{2}\right)
$$

where

$$
B_{k, j}=\sum_{p=0}^{k-j-1}(-1)^{k+j+1}\left(\begin{array}{c}
j+p \\
j
\end{array}\right)\left(\frac{k-1}{2}\right)^{p}\left(s_{k, j+p+1}+s_{k-1, j+p}\right), \quad 0 \leq j \leq k-1,
$$

and $\zeta(s, \alpha)$ is the familiar Hurwitz zeta-function $\sum_{r=0}^{\infty}(r+\alpha)^{-s}$ with $\alpha \in \mathbb{R}, \alpha>$ 0 .

Corollary. Taking $\delta_{1}=0, \delta_{2}=k-1$ we get for $Z_{k}(s)$

$$
(k-1) ! Z_{k}(s)=\sum_{l=0}^{\infty}(-1)^{l}\left(\frac{k-1}{2}\right)^{2 l}\left(\begin{array}{c}
-s \\
l
\end{array}\right) \sum_{j=0}^{k-1} B_{k, j} \zeta\left(2 s+2 l-j, \frac{k+1}{2}\right) .
$$

Theorem 2. The residue of the Minakshisundaram-Pleijel zeta function $Z_{k}(s)$ at the pole $s=\frac{k}{2}-n, n \in \mathbb{N}$, is

$$
\frac{1}{(k-1) !} \sum_{h=0}^{\frac{k}{2}-1} \sum_{\substack{l+h=n \\
l \geq 0}}(-1)^{l}\left(\frac{k-1}{2}\right)^{2 l}\left(\begin{array}{c}
n-\frac{k}{2} \\
l
\end{array}\right) B_{k, k-2 h-1} .
$$




\section{Proof of Theorem 1}

Applying (5) of [2] to $L_{k}(s)$ we have

(4)

$$
\begin{aligned}
&(k-1) ! L_{k}(s)= \sum_{l=0}^{\infty}(-1)^{l}\left(\frac{\delta_{2}-\delta_{1}}{2}\right)^{2 l}\left(\begin{array}{c}
-s \\
l
\end{array}\right) \\
& \cdot\left[\alpha_{k, 0} \zeta\left(2 s+2 l, \frac{k+1}{2}\right)\right. \\
&+\sum_{m=1}^{k-1} \sum_{j=0}^{m} \sum_{p=1}^{m} \alpha_{k, m} a_{p}^{m} P_{m-j}^{m}\left(m+1-p-\frac{k-1}{2}\right) \\
&\left.\cdot \zeta\left(2 s+2 l-j, p+\frac{k-1}{2}\right)\right]
\end{aligned}
$$

where, according the notation of [2]

$$
\begin{gathered}
a_{p}^{m}=\sum_{t=1}^{p}(-1)^{p-t}\left(\begin{array}{c}
m+1 \\
p-t
\end{array}\right) t^{m} \\
P_{m-j}^{m}(z)=\frac{1}{m !} \sum_{h=1}^{m+1} s_{m+1, h}\left(\begin{array}{c}
h-1 \\
j
\end{array}\right) z^{h-j-1} .
\end{gathered}
$$

If $k=2$, we get

$$
L_{2}(s)=\sum_{l=0}^{\infty}(-1)^{l}\left(\frac{\delta_{2}-\delta_{1}}{2}\right)^{2 l}\left(\begin{array}{c}
-s \\
l
\end{array}\right)\left(2 \zeta\left(2 s+2 l-1, \frac{3}{2}\right)\right) .
$$

Let us assume that $k \geq 3$. First we reduce the parameters $p+\frac{k-1}{2}$ of the Hurwitz zeta functions $\zeta\left(2 s+2 l-j, p+\frac{k-1}{2}\right)$ to $\frac{k+1}{2}$, so we have

$$
\begin{aligned}
(k-1) ! L_{k}(s)= & \sum_{l=0}^{\infty}(-1)^{l}\left(\frac{\delta_{2}-\delta_{1}}{2}\right)^{2 l}\left(\begin{array}{c}
-s \\
l
\end{array}\right) \\
& \cdot\left[\alpha_{k, 0} \zeta\left(2 s+2 l, \frac{k+1}{2}\right)\right. \\
& +\sum_{m=1}^{k-1} \sum_{j=0}^{m} \alpha_{k, m} a_{1}^{m} P_{m-j}^{m}\left(m-\frac{k-1}{2}\right) \zeta\left(2 s+2 l-j, \frac{k+1}{2}\right) \\
& +\sum_{m=2}^{k-1} \sum_{j=0}^{m} \sum_{p=2}^{m} \alpha_{k, m} a_{p}^{m} P_{m-j}^{m}\left(m+1-p-\frac{k-1}{2}\right) \\
& \left.\cdot\left[\zeta\left(2 s+2 l-j, \frac{k+1}{2}\right)-\sum_{t=0}^{p-2}\left(t+\frac{k-1}{2}\right)^{j-2 s-2 l}\right]\right] .
\end{aligned}
$$


We will prove that

(6) $\sum_{m=2}^{k-1} \sum_{j=0}^{m} \sum_{p=2}^{m} \sum_{t=0}^{p-2} \alpha_{k, m} a_{p}^{m} P_{m-j}^{m}\left(m+1-p-\frac{k-1}{2}\right)\left(t+\frac{k-1}{2}\right)^{j-2 s-2 l}=0$.

We rearrange the sums in left side of (6) as follows:

$$
\sum_{t=0}^{k-3} \sum_{m=t+2}^{k-1} \sum_{p=t+2}^{m} \sum_{j=0}^{m} \alpha_{k, m} a_{p}^{m} P_{m-j}^{m}\left(m+1-p-\frac{k-1}{2}\right)\left(t+\frac{k-1}{2}\right)^{j-2 s-2 l}=0 .
$$

Now, if $0 \leq t \leq k-3$ we obtain

$$
\begin{aligned}
\sum_{m=t+2}^{k-1} \sum_{p=t+2}^{m} \sum_{j=0}^{m} \alpha_{k, m} a_{p}^{m} \frac{1}{m !} \sum_{h=1}^{m+1} s_{m+1, h}\left(\begin{array}{c}
h-1 \\
j
\end{array}\right) & \cdot\left(m+1-p-\frac{k-1}{2}\right)^{h-1-j}\left(t+\frac{k-1}{2}\right)^{j} \\
= & \sum_{m=t+2}^{k-1} \sum_{p=t+2}^{m} \sum_{h=1}^{m+1} \frac{1}{m !} \alpha_{k, m} a_{p}^{m} s_{m+1, h} \\
= & \sum_{m=t+2}^{k-1} \sum_{p=t+2}^{m} \sum_{h=1}^{m+1} \frac{1}{m !} \alpha_{k, m} a_{p}^{m} s_{m+1, h}(m+1-p+t)^{h-1} \\
= & \left.\sum_{m=t+2}^{k-1} \sum_{p=t+2}^{m} \frac{1}{m !} \alpha_{k, m} a_{p}^{m} \frac{1}{(m+1-p+t)}\right)^{j} \\
& \cdot \sum_{h=1}^{m+1}(-1)^{h} s_{m+1, h}\left(p-\frac{k-1}{2}\right)^{h-1}(t-t-1)^{h}=0
\end{aligned}
$$

since

$$
\sum_{h=1}^{m+1}(-1)^{h} s_{m+1, h}(p-m-t-1)^{h}=\mathscr{R}(p-t-m-1, m+1)=0 .
$$

So (6) is proved.

Now we can write

(7)

$$
(k-1) ! L_{k}(s)=\sum_{l=0}^{\infty}(-1)^{l}\left(\frac{\delta_{2}-\delta_{1}}{2}\right)^{2 l}\left(\begin{array}{c}
-s \\
l
\end{array}\right) \sum_{j=0}^{k-1} B_{k, j} \zeta\left(2 s+2 l-j, \frac{k+1}{2}\right),
$$

where

(8) $B_{k, j}=\sum_{m=1}^{k-1} \sum_{p=1}^{m} \alpha_{k, m} a_{p}^{m} P_{m-j}^{m}\left(m+1-p-\frac{k-1}{2}\right), \quad 0<j \leq k-1$, 


$$
B_{k, 0}=\alpha_{k, 0}+\sum_{m=1}^{k-1} \sum_{p=1}^{m} \alpha_{k, m} a_{p}^{m} P_{m}^{m}\left(m+1-p-\frac{k-1}{2}\right) .
$$

From (8) and $\left(8^{\prime}\right)$ and [2] it follows that $B_{k, j}$ are independent on $\delta_{1}, \delta_{2}$. In order to obtain $B_{k, j}$ in a simpler form let us consider

$$
L(s)=\sum_{n=1}^{\infty} \frac{P_{k}(n)}{\left[n+\frac{k-1}{2}\right]^{2 s}} .
$$

So we have

$$
(k-1) ! L(s)=\sum_{j=0}^{k-1} B_{k, j} \zeta\left(2 s-j, \frac{k+1}{2}\right) .
$$

From

$$
(k-1) ! \sum_{n=1}^{\infty} \frac{P_{k}(n)}{\left[n+\frac{k-1}{2}\right]^{2 s}}=\sum_{j=0}^{k-1} B_{k, j} \zeta\left(2 s-j, \frac{k+1}{2}\right)
$$

we have

$$
(k-1) ! \sum_{n=1}^{\infty} \frac{P_{k}(n)}{\left[n+\frac{k-1}{2}\right]^{2 s}}=\sum_{n=1}^{\infty} \sum_{j=0}^{k-1} B_{k, j}\left(n+\frac{k-1}{2}\right)^{j}\left(n+\frac{k-1}{2}\right)^{-2 s}
$$

so that

$$
P_{k}(n)=\sum_{j=0}^{k-1} B_{k, j}\left(n+\frac{k-1}{2}\right)^{j}
$$

which gives, by the binomial theorem,

$$
\alpha_{k, m}=\sum_{j=m}^{k-1} B_{k, j}\left(\begin{array}{c}
j \\
m
\end{array}\right)\left(\frac{k-1}{2}\right)^{j-m}, \quad 0 \leq m \leq k-1 .
$$

From (9), solving a linear system, we get a formula for $B_{k, j}$ by far simpler than $(8)$ and $\left(8^{\prime}\right)$ :

$$
\begin{array}{r}
B_{k, j}=\sum_{p=0}^{k-j-1}(-1)^{k+j+1}\left(\begin{array}{c}
j+p \\
j
\end{array}\right)\left(\frac{k-1}{2}\right)^{p}\left(s_{k, j+p+1}+s_{k-1, j+p}\right), \\
0 \leq j \leq k-1 .
\end{array}
$$

So Theorem 1 is proved.

\section{Proof OF THEOREM 2}

Lemma. In the above corollary, $B_{k, j}=0$ if $j=0$ or if $j=k-2 h$ with $1 \leq h \leq\left[\frac{k}{2}\right]$. 
Proof. First step. From (10) we can immediately see that

$$
\begin{aligned}
B_{k, 0} & =\sum_{p=0}^{k-1}(-1)^{k+1}\left(\frac{k-1}{2}\right)^{p}\left(\left(s_{k-1, p}+s_{k, p+1}\right)\right) \\
& =\left[\mathscr{R}\left(\frac{1-k}{2}, k-1\right)+\frac{2}{1-k} \mathscr{R}\left(\frac{1-k}{2}, k\right)\right]=0 .
\end{aligned}
$$

Second step. If we put

$$
P_{n, t}(x)=\sum_{i=t}^{n}\left(\begin{array}{l}
i \\
t
\end{array}\right) s_{n, i} x^{i}, \quad Q_{n, t}(x)=\sum_{i=1}^{n}(-1)^{i+1} \frac{n !}{(n-i) !} P_{n-i, t}(x),
$$

we have

$$
Q_{n, t}(x)+Q_{n, t-1}(x)=\frac{n}{x} P_{n, t}(x) .
$$

In fact, starting from

$$
\begin{aligned}
Q_{n, t}(x)+ & Q_{n, t-1}(x)=\sum_{i=1}^{n}(-1)^{i+1} \frac{n !}{(n-i) !}\left[P_{n-i, t}(x)+P_{n-i, t-1}(x)\right] \\
& =\sum_{i=1}^{n}(-1)^{i+1} \frac{n !}{(n-i) !} \sum_{l=t-1}^{n-i}\left(\begin{array}{c}
l+1 \\
t
\end{array}\right) s_{n-i, l} x^{l} \\
& =\sum_{l=t-1}^{n-1}\left(\begin{array}{c}
l+1 \\
t
\end{array}\right) x^{l} \sum_{i=1}^{n-l}(-1)^{i+1} \frac{n !}{(n-i) !} s_{n-i, l} \quad \text { by using (1d) } \\
& =\sum_{l=t-1}^{n-1}\left(\begin{array}{c}
l+1 \\
t
\end{array}\right)\left[s_{n, l}-s_{n+1, l+1}\right] x^{l},
\end{aligned}
$$

(11) follows using (1a). By using (1a) iteratively in (10) we get

$$
B_{k, j}=2 P_{k-1, j}\left(\frac{k-1}{2}\right)-Q_{k-1, j}\left(\frac{k-1}{2}\right)
$$

so that, since by (11) with $n=k-1, x=\frac{k-1}{2}, t=j$

$$
2 P_{k-1, j}\left(\frac{k-1}{2}\right)=Q_{k-1, j}\left(\frac{k-1}{2}\right)+Q_{k-1, j-1}\left(\frac{k-1}{2}\right)
$$

we have

$$
B_{k, j}=Q_{k-1, j-1}\left(\frac{k-1}{2}\right) \text {. }
$$

Third step. Our thesis is equivalent to showing that

$$
Q_{k-1, j-1}\left(\frac{k-1}{2}\right)=0, \quad \text { if } j=k-2 h, h \in \mathbb{N} \text {. }
$$

By a simple direct computation, $Q_{k-1, k-3}=0$. By (12) it will be enough to prove that

$$
P_{k-1, j}\left(\frac{k-1}{2}\right)=P_{k-1, j-1}\left(\frac{k-1}{2}\right), \text { if } j=k-2 h-1>0, h \geq 1 .
$$


It is easy to see that

$$
P_{n, t}(x)=\frac{x^{t}}{t !} \frac{d^{t}}{d x^{t}} P_{n, 0}(x)
$$

So our thesis follows, taking $n=k-1, l=h$ if we prove prove that for $n \in \mathbb{N}$

$$
\frac{n}{2(n-2 l)} P_{n, 0}^{(n-2 l)}\left(\frac{n}{2}\right)=P_{n, 0}^{(n-2 l-1)}\left(\frac{n}{2}\right) \text {. }
$$

Observing that $P_{n, 0}(x)=x P_{n-1,0}(x-1)$ we have

$$
\begin{gathered}
P_{n, 0}^{(n-2 l)}\left(\frac{n}{2}\right)=(n-2 l) P_{n-1,0}^{(n-2 l-1)}\left(\frac{n}{2}-1\right)+\frac{n}{2} P_{n-1,0}^{(n-2 l)}\left(\frac{n}{2}-1\right), \\
P_{n, 0}^{(n-2 l-1)}\left(\frac{n}{2}\right)=(n-2 l-1) P_{n-1,0}^{(n-2 l-2)}\left(\frac{n}{2}-1\right)+\frac{n}{2} P_{n-1,0}^{(n-2 l-1)}\left(\frac{n}{2}-1\right),
\end{gathered}
$$

and (13) follows since

$$
P_{n-1,0}^{(n-2 l)}\left(\frac{n}{2}-1\right)=0, \forall l \in \mathbb{N} .
$$

In fact, $P_{n-1,0}(x)=G\left(x-\left(\frac{n}{2}-1\right)\right)$ with $G$ a function of parity opposite to $n$ 's one, and so having every derivatives of the same parity of $n$ 's one null. So Lemma is proved.

Proof of Theorem 2. From the Lemma and Theorem 1 we can easily deduce the following facts:

$Z_{k}(s)$ is a meromorphic function with a simple pole at $s=\frac{k}{2}$.

If $k$ is even, $Z_{k}(s)$ has simple poles at most at positive integers $s=$ $\frac{k}{2}-n, n \in \mathbb{N}$.

If $k$ is odd, $Z_{k}(s)$ has simple poles at most at rationals $s=\frac{k}{2}-n, n \in$ $\mathbb{N}$.

Furthermore, we can give the following formula for the residues of $Z_{k}(s)$ at $s_{0}=\frac{k}{2}-n_{0}, n_{0}$ a nonnegative integer, recalling that the residue of the Hurwitz zeta function at its pole is 1 :

$$
\frac{1}{(k-1) !} \sum_{h=0}^{\frac{k}{2}-1} \sum_{\substack{l+h=n_{0} \\
l \geq 0}}(-1)^{l}\left(\frac{k-1}{2}\right)^{2 l}\left(\begin{array}{c}
n_{0}-\frac{k}{2} \\
l
\end{array}\right) B_{k, k-2 h-1}
$$

So Theorem 2 is proved.

Remarks. 1. If $k$ is odd, $Z_{k}(s)$ vanishes at $s=-n, n \in \mathbb{N}$. In fact, from

$$
(k-1) ! Z_{k}(-n)=\sum_{l=0}^{n}(-1)^{l}\left(\frac{k-1}{2}\right)^{2 l}\left(\begin{array}{l}
n \\
l
\end{array}\right) \sum_{j=1}^{k-1} B_{k, j} \zeta\left(-2 n+2 l-j, \frac{k+1}{2}\right),
$$

we note that

$$
\zeta\left(-2 n+2 l-j, \frac{k+1}{2}\right)=\zeta(-2 n+2 l-j)-\sum_{t=1}^{\frac{k+1}{2}-1} \frac{1}{t^{-2 n+2 l-j}}
$$


where $\zeta(s)$ is the Riemann zeta function which vanishes at negative even integers, so we have

$$
\begin{aligned}
& \sum_{j=0}^{k-1} B_{k, j} \sum_{t=1}^{\frac{k+1}{2}-1} \frac{1}{t^{-2 n+2 l-j}} \\
& \quad=\sum_{j=1}^{k-1} \sum_{m=1}^{k-1} \sum_{p=1}^{m} \alpha_{k, m} a_{p}^{m} P_{m-j}^{m}\left(m+1-p-\frac{k-1}{2}\right) \sum_{t=1}^{\frac{k+1}{2}-1} \frac{1}{t^{-2 n+2 l-j}}=0,
\end{aligned}
$$

by the same argument used to get (7).

2. From $B_{k, j}=0$ if $j=k-2 h$ or if $j=0$ we deduce easily the following relations among the Stirling numbers

$$
\sum_{j=k-2 h}^{k-1}\left(\begin{array}{c}
j \\
k-2 h
\end{array}\right)\left(\frac{k-1}{2}\right)^{j}\left(s_{k, j+1}+s_{k-1, j}\right)=0, \quad 1 \leq h \leq \frac{k}{2} .
$$

Moreover, we also have from (9)

$$
(k-1) !=\sum_{j=0}^{k-1} B_{k, j}\left(\frac{k-1}{2}\right)^{j} .
$$

3. The Minakshisundaram-Pleijel zeta function $L_{k}(s)$ of $k$-dimensional real projective space $\mathbb{P}^{k}$ takes the form, in $\Re e(s)>\frac{k}{2}$,

$$
L_{k}(s)=\sum_{n=1}^{\infty} \frac{P_{k}(2 n)}{[2 n(2 n+k-1)]^{s}} .
$$

It is easy to see that

$$
4^{s}(k-1) ! L_{k}(s)=\sum_{l=0}^{\infty}(-1)^{l}\left(\frac{k-1}{4}\right)^{2 l}\left(\begin{array}{c}
-s \\
l
\end{array}\right) \sum_{j=0}^{k-1} 2^{j} B_{k, j} \zeta\left(2 s+2 l-j, \frac{k+3}{4}\right)
$$

where $B_{k, j}$ are the same as for $\mathbb{S}^{k}$.

\section{REFERENCES}

1. M. Berger, P. Gauduchon, and E. Mazet, Le spectre d'une variété riemannienne, SpringerVerlag, New York, 1971.

2. E. Carletti and G. Monti Bragadin, On Dirichlet series associated with polynomials, Proc. Amer. Math. Soc. 121 (1994), 33-37.

3. P. Gilkey, Invariance theory. the heath equation and the Atiyah-Singer index theory, Publish or Perish, Cambridge, MA, 1984.

4. C. Jordan, Calculus of finite differences, Chelsea, New York, 1965.

5. S. Minakshisundaram and A. Pleijel, Some properties of the eigenfunctions of the Laplace operator on Riemannian manifolds, Canad. J. Math. 1 (1949), 242-256.

Dipartimento di Matematica, Universita di Genova, Via L. B. Alberti 4, 16132 Genova, ITALY 